\title{
Satellite Handover Techniques for LEO Networks
}

\author{
E. Papapetrou, S. Karapantazis, G. Dimitriadis, F-N. Pavlidou
}

\begin{abstract}
Low earth orbit (LEO) satellite constellations could play an important role in future mobile communication networks due to their characteristics, such as global coverage and low propagation delays. However, because of the non-stationarity of the satellites, a call may be subjected to handovers, which can be cell or satellite handovers. Quite many techniques have been proposed in the literature dealing with the cell handover issue. In this paper, a satellite handover procedure is proposed, that investigates and exploits the partial satellite diversity (namely, the existing common coverage area between contiguous satellites) in order to provide an efficient handover strategy, based always on a tradeoff of the blocking and forced termination probabilities for a fair treatment of new and handover calls. Three different criteria were examined for the selection of a satellite. Each one of them could be applied either to new or handover calls, therefore we investigated nine different service schemes. A simulation tool was implemented in order to compare the different service schemes and simulation results are presented at the end of the paper.
\end{abstract}

\section{Introduction}

Future communication networks aim at providing high quality services with global coverage. Thus, the satellite component is foreseen to be widely utilized. Satellite constellations can be fairly efficient in many ways. They are particularly effective when used to provide two categories of service, broadcasting and multicasting, whereas they could also be considered as a backbone for terrestrial communication systems. For these reasons, the 3G mobile systems worldwide comprise of interworking terrestrial and satellite components (i.e. UMTS, IMT2000). Regarding real-time and interactive services, Low Earth Orbit (LEO) satellite constellations emerge as the most convenient solution because of the low propagation delays they provide [13]. Several LEO constellations have been proposed in the literature (Globalstar, Iridium, Ellipso etc.), while the operation of the Iridium system has offered a very good experience for the study of the critical performance issues of these systems.

In LEO systems the visibility period of a satellite can be as short as 5 min due to the continuous rotation of satellites, while current common practice is to divide the satellite footprint in many cells (using multibeam arrays), in order to enhance frequency reuse policies (fig. 1). This leads to a significant probability of service interruption and consequently, the hand-over mechanism becomes of great importance for the overall performance of the system. That is, in LEO in parallel to the classic performance criteria (blocking probability $P_{b}$, delay $D$, throughput $T$ etc) the forced termination probability $\left(P_{f}\right)$ is a crucial parameter, as is the case in land mobile systems. There are two types of handover events, the cell handover and the satellite handover. The former refers to the transfer of an ongoing call from one cell to the next one in the same satellite footprint while the latter describes the transfer of an ongoing call from a satellite to another one (fig.1).

Quite many studies have been carried out on the issue of cell handover, investigating channel allocation policies for new and handover calls mainly through fixed channel allocation (FCA) techniques. In [1] different queuing policies for handover requests were proposed. The handover requests, queued up to a maximum time interval (which is a function of the overlapping area of contiguous cells), are served in a first-inputfirst-output (FIFO) scheme or in a last useful instant (LUI) scheme (that is, a handover request is queued ahead of any other requests already in the queue that have a longer residual queuing time).A new call is always admitted in the network if an available channel exists in the current cell. New calls generated in the overlapping area of adjacent cells are immediately addressed to the destination cell in order to avoid an immediate handover. A call is forced into termination if the handover request is not served within the queuing time in the current cell.

In [2] a guaranteed handover service was proposed. According to this method a new call is admitted in the network only if there is an available channel in the current cell and simultaneously in the first transit 


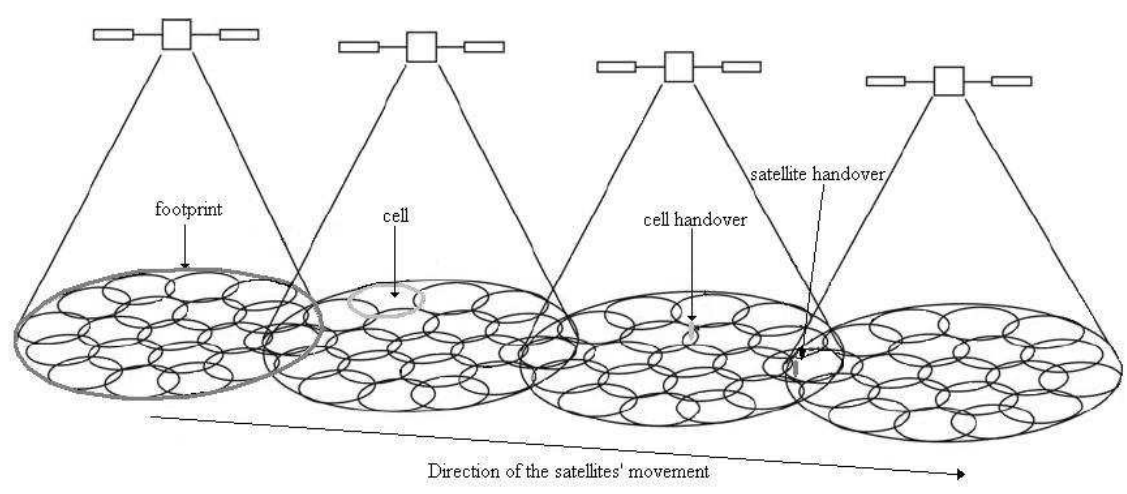

Figure 1: Footprints of LEO satellites

cell. When the first handover occurs a channel reservation request is issued to the next candidate transit cell and so on. If all channels of the next cell are busy, the request is queued in a list in a FIFO discipline until the occurrence of the next handover. This technique provides zero $P_{f}$ but at the cost of unacceptably high values of $P_{b}$ due to very early channel reservation.

In [3] a connection admission control strategy for cell handover was studied in detail. A geographical connection admission control $(G C A C)$ algorithm was introduced in addition to an adaptive dynamic channel allocation ( $A D C A)$ scheme. According to the $G C A C$ algorithm, the future forced termination probability for a new call and for the existing calls is estimated as a function of user location and it is checked if it is below a predefined level. Upon this check the $G C A C$ algorithm determines whether the new call is accepted or not.

In [4] a dynamic Doppler based handover prioritization technique (DDBHP) has been proposed. This method takes advantage of the Doppler effect in order to estimate the terminal location and to reserve channels at an appropriate time in the servicing and forthcoming satellite. The term appropriate time defines a time interval (time threshold $t_{T H}$ ), prior to the handover occurrence, during which resource allocation activities should be completed. The instant prior to the handover of the terminal, on which a channel reservation request is sent to the forthcoming satellite, is defined by $t_{T H}$.

Concluding, while in the literature the issue of cell handover is quite well investigated, there is a lack of studies for satellite handover techniques, due to the fact that the cell handover is more frequent than the satellite handover. However, satellite handover is also a really crucial parameter in LEO satellite diversity based systems. Contrary to the cell handover at which the user has no choice of selection, in the satellite handover, the user can select among different satellites. Furthermore the user first selects the servicing satellite and then is served by the cell that covers him. So, separate algorithms are needed to treat the satellite handover and the cell handover. The satellite handover algorithm should target to select the most suitable satellite in means of $P_{b}, P_{f}$ and quality of communication while the cell handover algorithm should assure that the call will not be dropped in the time between two handovers. Therefore, a well studied satellite handover technique could avoid wasting bandwidth while it could also fulfill the quality constraints of the supported services in means of $P_{b}$ and $P_{f}$.

Study [6] covers the satellite diversity issue, but not aiming at investigating it in detail, rather to estimate the end-to-end path delay of a call in the space segment. In [7], two handover algorithms are proposed for systems with satellite diversity; (i) a hard handover scheme with different power thresholds for adding/dropping the satellites involved in the handover process and (ii) a hybrid channel adaptive selective scheme $(C A S D)$ - which uses the two - threshold concept of the hard operation and at the same time is generic as it can operate in hard or soft handover. However, in this study only the case in which a call is dropped due to power limitations is considered. Nevertheless, a call could also be forced into termination if there is not a free channel in the forthcoming satellite. Furthermore, a study investigating the performance of each algorithm for different values of traffic load would be beneficial.

In many proposed satellite networks (Teledesic), contiguous satellites share common coverage areas on the surface of the earth (partial satellite diversity). Partial satellite diversity means that there may be users covered only by one satellite. Satellite diversity (or partial satellite diversity) it is welcome because it can support drastically efficient bandwidth utilization techniques and a very flexible system operation 
for providing QoS services in future systems. Thus a thorough investigation of constellations with partial satellite diversity is quite beneficiary for an efficient performance of such systems.

In this paper the satellite handover issue is studied in depth. The proposed technique aims at handling the satellite handover issue in an optimum way and therefore providing users with high quality of service at quite low forced termination probability while it avoids wasting the limited and costly satellite bandwidth. In addition, our technique fulfills the QoS limitations even for heavy traffic conditions. We base our analysis on the $D D B H P$ procedure proposed in [4], as it seems to offer a suitable tradeoff between blocking and forced termination probabilities, modifying it for the case of satellite handover. We focus our study on a network that resembles the Boeing design of the Teledesic system (288 satellites), because it presents partial satellite diversity and the specifications of this design are quite well defined. We also examined our technique in an Iridium-like network in order to see if there are any differences when applying the proposed technique in a system with a different geometry. Considering the common areas that satellites in different orbital planes share, the user can select between more than one satellites and thus we have to define criteria for that selection. We propose and evaluate three criteria, each of them being applied either to new or handover calls. Consequently, we result in nine different service schemes and we investigate the overall system performance for each one of them. Throughout in our study we neglect the cell handover since we like to focus on the satellite handover. Of course, we should examine the common phenomenon of cell and satellite handover, but for the moment this is out of the scope of our study.

The remaining of the paper is organized as follows. Section 2 describes the mobility model and the proposed technique in detail. The simulation framework and the performance evaluation of the system for the different service schemes are presented in section 3. Finally, section 4 summarizes the results and concludes.

\section{Mobility modelling and channel reservation procedures}

In non-GEO satellite constellations the visibility period of a satellite can be rather small. Future satellite networks should be compatible with terrestrial systems (S-UMTS), therefore voice will not be the sole service they will provide. Interactive multimedia IP services are expected to be of utmost importance (and obviously, for this type of services quite many satellite handovers will occur). Teledesic will definitely support IP services. Although this system does not provide always dual satellite coverage, its constellation design presents partial satellite diversity, and therefore, provides the possibility for satellite handover between satellites in different orbital planes.

\subsection{Mobility model}

In Teledesic adjacent satellite footprints share common areas on the earth surface (partial satellite diversity) as it is shown in fig. 2. We consider an approximate two-dimensional design where the satellite footprints have orthogonal shape (fig. 3). This model is valid as far as the following assumptions are met.

- Users are considered fixed on the earth surface, while satellites move with a constant speed $V_{\text {sat }}$. This is true if we take into account that terminals in very fast vehicles move with a velocity of $80 \mathrm{~m} / \mathrm{sec}$ at most, whereas the satellite velocity (for LEO constellations) is approximately $7400 \mathrm{~m} / \mathrm{sec}$. Furthermore, we do not take into consideration the rotation of the earth.

- A user can select only between satellites in different orbital planes at call setup. We do not consider the case wherein the user can select between contiguous satellites in the same orbital plane, because in that case the user should always select the following satellite in order to avoid an immediate handover. With regard to fig.3, the gray area between satellites 7 and 10 presents the common area between contiguous satellites in the same orbital plane.

- Terminals are uniformly distributed on the earth surface and in each satellite footprint.

- The system is a polar and not an inclined network. This is true for Teledesic and Iridium.

Fig. 3 illustrates the service procedure of the system. If user A generates a new call, he can be served either by satellite 3 or satellite 2 . Regarding the first option, he can again select between two satellites $(6,5)$. However, user B can be served only by satellite 3 and will be handed-over to satellite 6 . We see thus that there is a quite flexible selection environment in the system. 
As previously said, the proposed algorithm is based on the $D D B H P$ technique [4], which makes use of the Doppler effect to avoid early reservation of channels and favors low blocking probability. The application of a Doppler-based positioning technique for users in a footprint has been examined in several proposals in the literature $[4,12,13]$ and has been proved to be an efficient and low-complexity method for predicting handover requests and reserving channels into the interval defined by $t_{T H}$. Describing briefly $D D B H P$ we note that by measuring the Doppler shift at two different time instants, it is possible to estimate the location of the user terminal and the time at which the handover will take place (station monitoring). Furthermore, by knowing the position of other satellites, the servicing satellite is able to select the possible forthcoming satellites for relaying the calls. This is an important feature of the $D D B H P$ technique since the servicing satellite is not always the following one in the same orbital plane.

\subsection{Channel Reservation Procedures}

According to the proposed algorithm, a new call is admitted in the network if an available channel is found in the current satellite. However, if the location of the users terminal indicates that a handover will occur in a time interval less than $t_{T H}$ then a channel is simultaneously reserved at the satellite selected for the first handover, otherwise the call is blocked. After the call is admitted in the network, station monitoring is activated by the servicing satellite. The selection of the next servicing satellite is based on three criteria described below.

Regarding subsequent handovers, a channel-reservation request is sent to the next satellite at a time defined by $t_{T H}$ before the handover occurrence. If an available channel is not found in the meantime, then the call is forced into termination. The selection of $t_{T H}$ is crucial. High values of $t_{T H}$ lead to small values of forced termination probabilities compared to forced termination probabilities for small values of $t_{T H}$, but blocking probabilities are unacceptably high due to early reservation of resources. On the contrary, small values of $t_{T H}$ result to smaller values of blocking probabilities. Apparently, different values of $t_{T H}$ define different quality of service levels. A study on the determination of the range of $t_{T H}$ is given in the following Section.

For the selection of the next servicing satellite we propose the following three criteria.

1. Maximum service time

According to this criterion, the user will be served by the satellite that offers the maximum service period. This criterion aims at minimizing the number of handovers and therefore achieving low forced termination probabilities.

2. Maximum number of free channels

According to this criterion, the user will be served by the satellite with the maximum number of free channels. The aim in this case is to achieve a uniform distribution of the telecommunication traffic in the celestial network. Thus, new or handover calls experience the same blocking or forced termination probabilities in every satellite regardless their location, avoiding, therefore, overloaded satellites.

3. Minimum distance

According to this criterion, the user will be served by the closest satellite. This criterion aims at avoiding link failures depending on the distance between the user terminal and the satellite. As far as we know there is no known probability function that describes link failure occurrences. Nevertheless, simulation results will show that it is worth examining this criterion.

Since the criteria can be applied to both new and handover calls, we result in nine different service schemes that are shown in Table $I$.

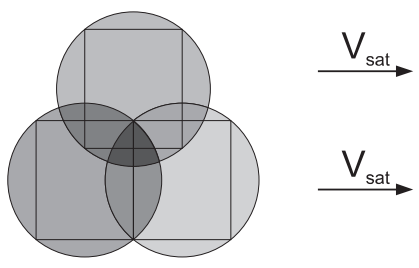

Figure 2: Partial satellite diversity

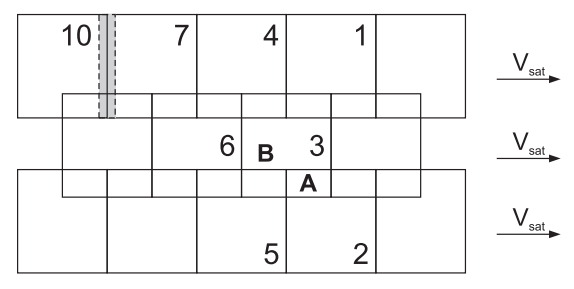

Figure 3: Mobility model 
Table 1: Service Schemes

\begin{tabular}{|l|l|l|}
\hline Service scheme & New Calls criterion & Handover criterion \\
\hline TT scheme & Max service Time & Max service Time \\
\hline CC scheme & Max number of free Channels & Max number of free Channels \\
\hline DD scheme & Min Distance & Min Distance \\
\hline TC scheme & Max service Time & Max number of free Channels \\
\hline TD scheme & Max service Time & Min Distance \\
\hline CT scheme & Max number of free Channels & Max service Time \\
\hline CD scheme & Max number of free Channels & Min Distance \\
\hline DT scheme & Min Distance & Max service Time \\
\hline DC scheme & Min Distance & Max number of free Channels \\
\hline
\end{tabular}

Investigating the reservation techniques in detail we notice that according to the proposed mobility model the number of the possible servicing satellites can be two at most (the case of user A in fig. 3). The new call admission procedure has as follows. A new call will first check if there is a free channel in the satellite indicated by the criterion used for the access procedure (assume that this satellite is satellite number 3 ). If no channel is found, then it will check the second satellite (satellite number 2). The reservation procedure for handover calls has as follows. At a handover request the servicing satellite decides on the next possible servicing satellite according to the criterion used. We consider again the case of two satellites covering the user area (we assume that user A was initially served by satellite 3). At the time of the handover occurrence, the selected satellite is checked (assume that it will be satellite 6). If a channel has been reserved in the meantime, then the call is handed-over to this satellite and if a channel has been reserved in satellite 5, it is released; otherwise the request is deleted from the queue. If a channel has not been reserved in satellite 6 , the request is deleted from the queue and satellite 5 is checked. If a channel has been reserved, the call is handed-over to this satellite, otherwise is forced into termination and the request is deleted from the queue.

If a call is terminated in $t_{T H}$, the reserved channel in each one of the forthcoming satellites is released. If there is no reserved channel in a satellite, the request is just deleted from the queue of this satellite. The messages for a channel reservation are sent to the forthcoming satellites through inter-satellite links (ISLs). A general flow chart of the implemented algorithms is presented in fig. 4

\section{Performance Evaluation}

\subsection{Simulation Parameters}

A simulation tool has been developed in $\mathrm{C}++$ and extended runs for different system configurations provided reliable and interesting information on the system performance. We examined the performance of each one of the nine service schemes proposed in Table $I$ in a typical low earth orbit constellation that resembles the geometry of the Teledesic system (Boeing design 288 satellites). The specifications of this system are presented in Table II. According to this design, contiguous satellites in different orbital planes share a common area of about $13 \%$ of the footprint total area. For the simulation runs we adopted the mobility model mentioned in Section 2. We simulated 4 orbits with 6 satellites in each one. Users from the first satellite could be handed-over to the sixth satellite. Furthermore, we applied the parameters of Table III. $t_{F}$ defines the maximum time that a mobile user can stay in a satellite footprint. Each mobile user generates calls according to a Poisson distribution function with a rate $\lambda_{\text {user }}$, while $T_{\text {call }}$ is the average call duration.

Moreover, we examined different values of the time threshold $t_{T H}$ in order to see its influence on blocking and forced termination probabilities. We also tested the performance of the schemes for different values of the load per footprint.

\subsection{Simulation Results}

As mentioned before, different values of $t_{T H}$ define different quality of service levels. Fig. 5 and 6 present blocking and forced termination probabilities for a network that resembles the Teledesic system $\left(\frac{\text { common coverage area }}{\text { footprint's area }}=13 \%\right.$ ) and for service schemes that use the same criterion both for the access and 


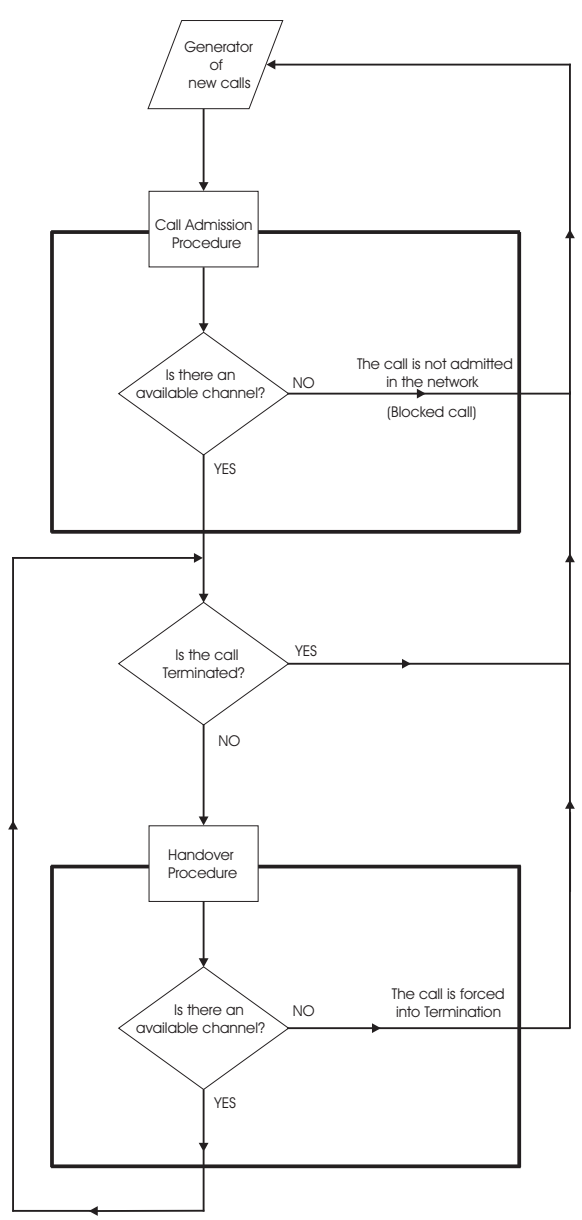

Figure 4: Flowchart

Table 2: Teledesic's specifications

\begin{tabular}{|l|l|}
\hline Number of planes & 12 \\
\hline Number of satellites per plane & 24 \\
\hline Satellite altitude & $1375 \mathrm{Km}$ \\
\hline Inclination & $85^{\circ}$ \\
\hline Acceptable minimum elevation angle & $40^{\circ}$ \\
\hline
\end{tabular}

Table 3: Simulation parameters

\begin{tabular}{|l|l|}
\hline Footprint Length & $1667.6 \mathrm{Km}$ \\
\hline$T_{f}$ (time in a footprint) & $4.71 \mathrm{~min}$ \\
\hline$V_{\text {sat }}$ & $7.169 \mathrm{Km} / \mathrm{sec}$ \\
\hline Channels per Satellite & 10 \\
\hline Users per footprint & 100 \\
\hline$T_{\text {call }}$ (call duration) & $180 \mathrm{sec}$ \\
\hline Load per footprint & $8 \mathrm{Erlang}$ \\
\hline$\lambda_{\text {user }}\left(\right.$ arrival rate $10^{-4}$ calls/sec) & 4.44 \\
\hline
\end{tabular}

the handover procedure. As we expected, the higher $t_{T H}$ is, the higher blocking probabilities are. On the contrary, as $t_{T H}$ increases, a drop in forced termination probabilities is observed. We also observe that the 
CC and the TT scheme perform better than the DD scheme. Moreover, they seem to have a similar performance. The TT scheme presents better blocking probabilities, whereas the CC scheme performs better as far as forced termination probabilities are concerned. However, we can say that the CC scheme performs slightly better, because forced termination calls are less desirable from the user's point of view than blocked calls.

Fig. 7 and 8 illustrate the performance of the other six schemes of Table I, for the same network. The results are fairly interesting. The best performance is obtained for the CT scheme, while the worst for the CD scheme, both for new and handover calls. The differences among the schemes are more obvious in blocking probabilities than in forced termination probabilities. At this point, we should say that several simulation runs showed that the DC, the DT and the DD schemes perform better for smaller values of the common coverage area but only regarding blocking probabilities, whilst all the other schemes present lower probabilities for higher values of the common coverage area. We also see that only the CT scheme performs better than the CC and the TT schemes and therefore, CT scheme seems to be the best case among all experiments.

Simulation runs for other values of the telecommunication load (2, 4 and 6 Erlang) showed that the CT scheme performs better than any other scheme for all the different values of load. Considering that each satellite has 10 channels, 2 (4 or 6) Erlang means that all the channels of the satellite are reserved for the $20 \%(40 \%$ or $60 \%)$ of the simulation time interval. Also, the performance of the CC scheme seemed to be very close to the performance of the CT scheme. Fig. 9 and 10 present the performance of all the schemes for different values of load and for $t_{T H} / t_{F}=6 \%$. For high values of load (6 and 8 Erlang) there is a considerable difference in the performances of the schemes. The differences among the performances are obvious both in $P_{b}$ and in $P_{f}$, that is to say, the best scheme (CT scheme) has $P_{b}=0.1275$ and $P_{f}=0.0098$ while the worst scheme (CD scheme) has $P_{b}=0.1787$ and $P_{f}=0.01685$ (for load=8 Erlang).

Of course in a realistic system we have always an overlapping of satellite footprints, something that we try to avoid for interference, waste of bandwidth and economical reasons. But since it exists we provide data in fig. 11 and 12 on the influence of different values of overlapping on blocking and forced termination probabilities for a Teledesic-like system applying the CT scheme. An increment in the common area between contiguous satellites can be achieved either by increasing the altitude of the orbits or by adding another orbital plane. Simulation runs for all the schemes showed that the bigger the common coverage area is, the better the scheme performs. We should notice that the case of $25 \%$ common coverage area is the marginal case of full satellite diversity, where all users are covered by two satellites.

Examining the evolution of Teledesic [16], a vital parameter for the success of a system is the constructive and operation cost, and therefore, future designs of non-GEO satellite systems tend to decrease the number of the satellites by increasing the altitude of the orbits. So, we checked the schemes on an Iridium-like system, resulting essentially to an analogous performance for each one of the schemes. Table IV presents the specifications of this system. We would not purpose on a quantitative analysis of the results of the two networks (Teledesic-like and Iridium-like), rather than we wanted to examine if there was a difference in the quality ranking of the schemes. Again the CT scheme presented the best performance. Fig. 13, 14, 15 and 16 show the performance of the schemes for this system. All the simulation parameters were still the

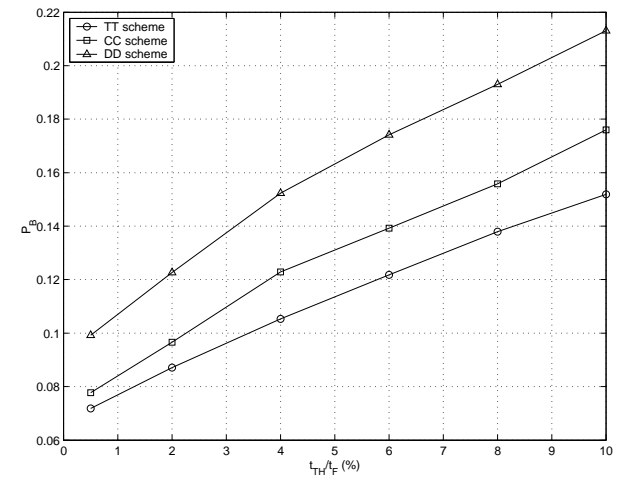

Figure 5: $P_{b}$ for Teledesic if the same selection criterion is applied to the access and handover procedure

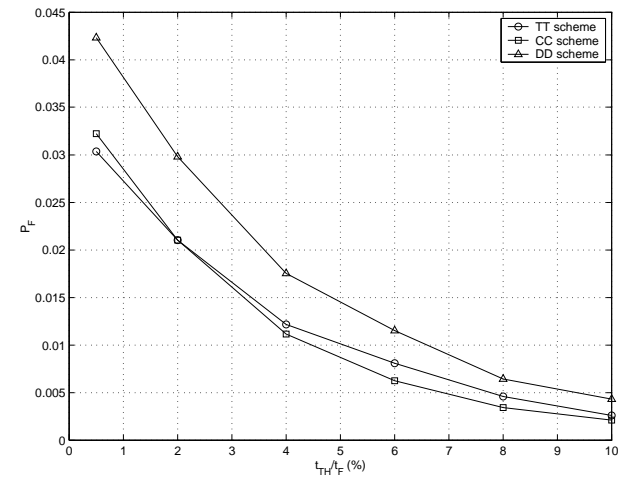

Figure 6: $P_{f}$ for Teledesic if the same selection criterion is applied to the access and handover procedure 


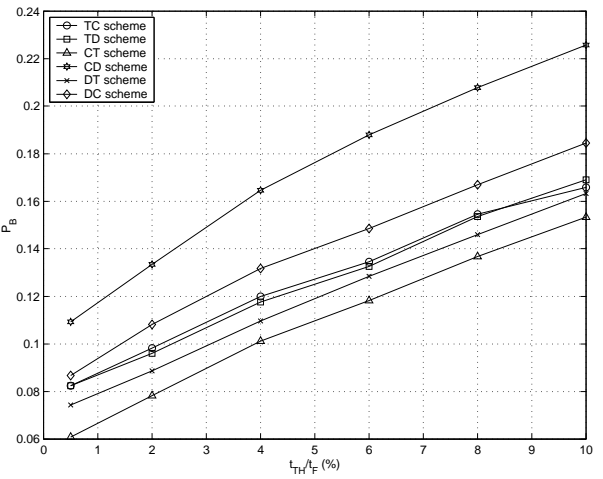

Figure 7: $P_{b}$ for Teledesic if different selection criteria are applied to the access and handover procedure

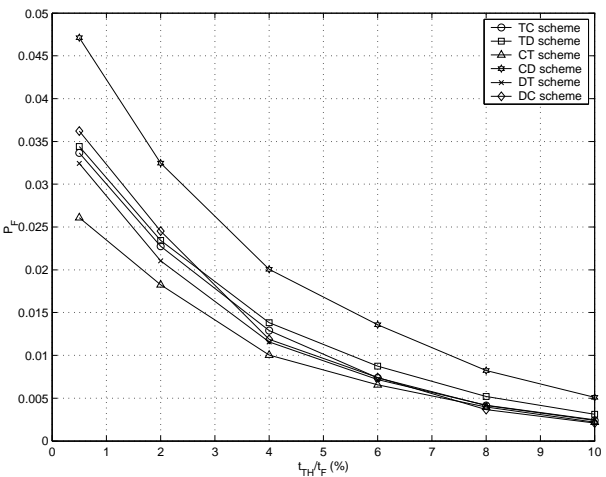

Figure 8: $P_{f}$ for Teledesic if different selection criteria are applied to the access and handover procedure

same except for the following: Footprint length $=3638.53 \mathrm{Km}, t_{F}=9.18 \mathrm{~min}, V_{\text {sat }}=7.4458 \mathrm{Km} / \mathrm{sec}$.

Table 4: Iridium's specifications

\begin{tabular}{|l|l|}
\hline Number of planes & 6 \\
\hline Number of satellites per plane & 11 \\
\hline Satellite altitude & $780 \mathrm{Km}$ \\
\hline Inclination & $86.4^{\circ}$ \\
\hline Acceptable minimum elevation angle & $8^{\circ}$ \\
\hline
\end{tabular}

The obtained results are quite promising and illustrate that an effective design of a partial satellite diversity constellation is possible at a low complexity algorithm resulting in a favorable allocation of resources and satisfactory QoS provision.

\section{Conclusions}

In this paper a prioritization technique that is based upon the $D D B H P$ technique for handling the satellite handover issue has been proposed. The proposed technique aims at fulfilling the QoS limitations even for heavy traffic conditions, while it avoids wasting the limited satellite bandwidth. It takes into account the partial satellite diversity that future LEO networks will present and it defines three different criteria for the selection of a satellite. The three different criteria resulted in nine different service schemes and we tested

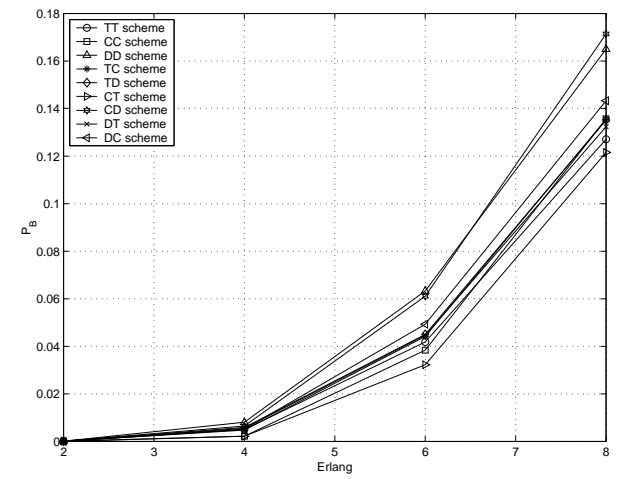

Figure 9: $P_{b}$ for Teledesic and for different loads

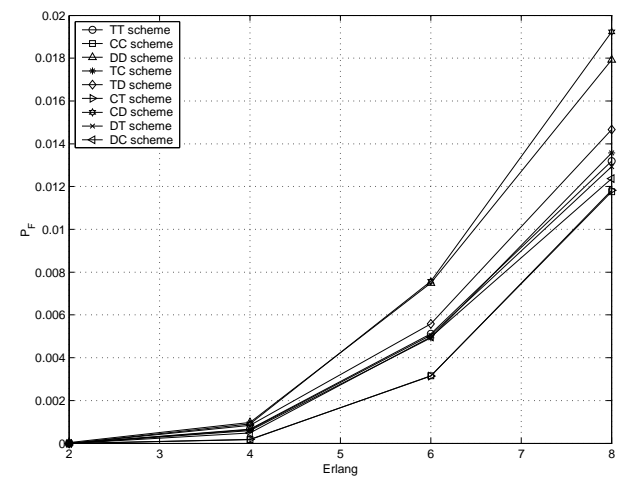

Figure 10: $P_{f}$ for Teledesic and for different loads 


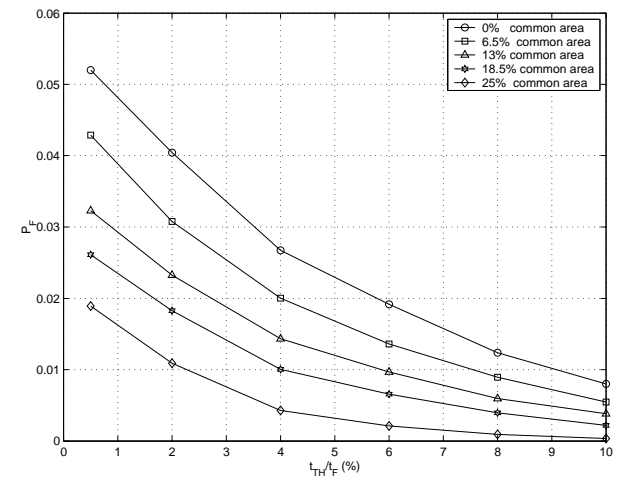

Figure 11: $P_{b}$ for the CT scheme and for different values of the common coverage area

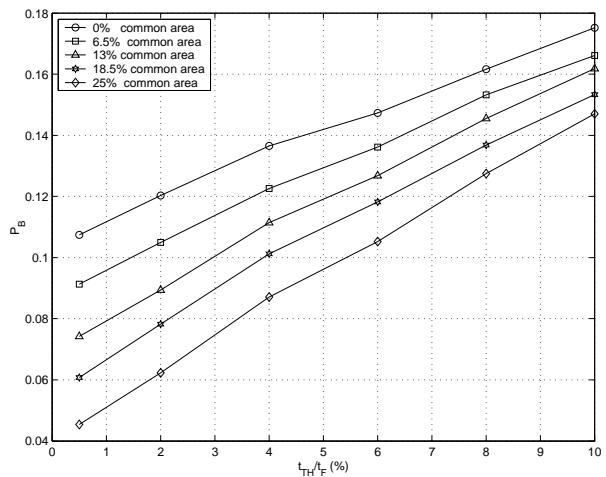

Figure 12: $P_{f}$ for the CT scheme and for different values of the common coverage area

these schemes in two different networks in order to derive the scheme with the best performance. Obviously, different criteria and different values of the time threshold can be used by users in different areas either for the access or the handover procedure, always according to the prospective telecommunication load.

\section{References}

[1] Enrico Del Re, Romano Fantacci, Giovanni Giambene. Different Queuing Policies for Handover Requests in Low Earth Orbit Mobile Satellite Systems. IEEE Transactions on Vehicular Technology, vol. 48, No. 2, March 1999.

[2] Gerard Maral, Joaquin Restrepo, Enrico Del Re, Romano Fantacci, Giovanni Giambene. Performance Analysis for a Guaranteed Handover Service in a LEO Constellation with a Satellite-Fixed Cell System. IEEE Transactions on Vehicular Technology, vol. 47, No. 4, November 1998.

[3] Sungrae Cho, Ian F. Akyildiz, Michael D. Bender, Huseyin Uzunalioglu. A New Connection Admission Control for Spotbeam Handover in LEO Satellite Networks. Kluwer Academic Publishers, Wireless Networks, Vol. 8, Issue 4 (July 2002).

[4] E. Papapetrou, E. Stathopoulou, F.-N. Pavlidou. Supporting QoS over Handovers in LEO Satellite Systems. Mobile \&6 Wireless Telecommunications Summit 2002, 17-19 June 2002/ Thessaloniki Greece.

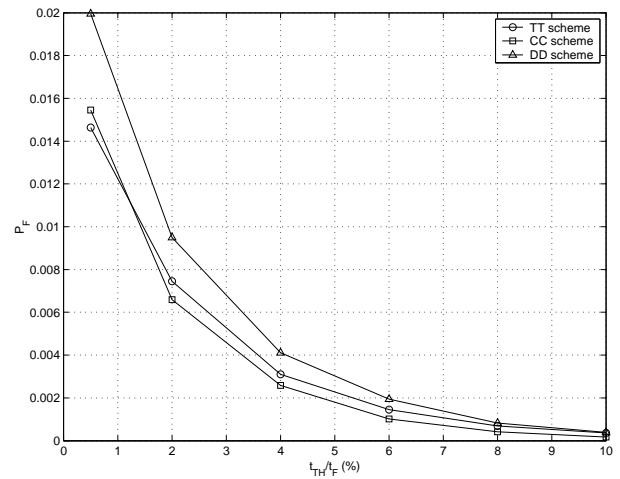

Figure 13: $P_{b}$ for Iridium if the same selection criterion is applied to the access and handover procedure

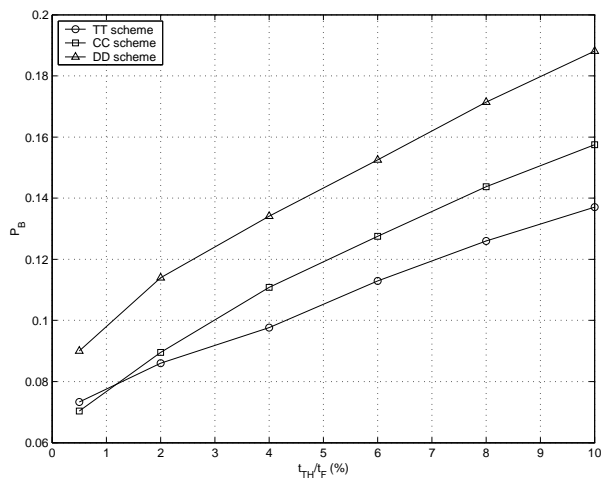

Figure 14: $P_{f}$ for Iridium if the same selection criterion is applied to the access and handover procedure 


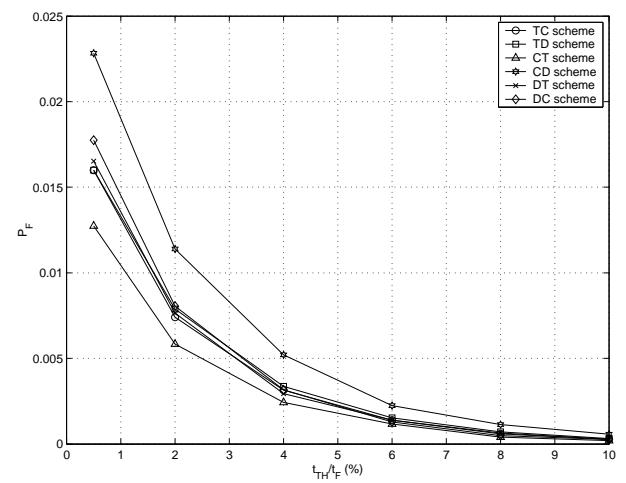

Figure 15: $P_{b}$ for Iridium if different selection criteria are applied to the access and handover procedure

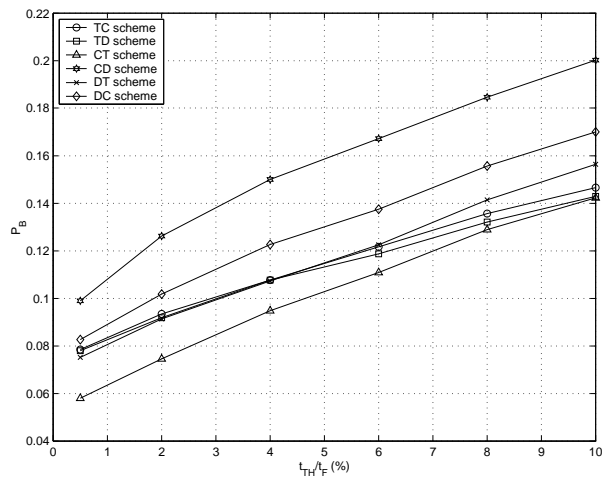

Figure 16: $P_{f}$ for Iridium if different selection criteria are applied to the access and handover procedure

[5] Enrico Del Re, Romano Fantacci, Giovanni Giambene. Characterization of user mobility in low earth orbit mobile satellite systems. Kluwer Academic Publishers, Wirelless Networks, Vol. 6, Issue 3 (July 2000)

[6] Lloyd Wood, George Pavlou, Barry Evans. Managing diversity with handover to provide classes of service in satellite constellation networks. Proceedings of the 19th AIAA International Communication Satellite Systems Conference (ICSSC'01), Toulouse, France, April 2001

[7] Maria Gkizeli, Rahim Tafazolli, Barry G. Evans. Hybrid Channel Adaptive Handover Scheme for NonGEO Satellite Diversity Based Systems. IEEE Com Letters, Vol.5 No.7, July 2001

[8] Maria Gkizeli, Rahim Tafazolli, Barry G. Evans. Performance Analysis of Handover Mechanisms for Non-Geo Satellite Diversity Systems. Proc. IEEE GLOBECOM 2001, Vol.4, pp. 2744-2748

[9] Suresh Kalyanasundaram, Edwin K.P. Chong, Ness B. Shrof. An Eficient Scheme to Reduce Handoff Dropping in LEO Satellite Systems. Kluwer Academic Publishers, Wireless Networks, Vol. 7, Issue 1, p. $75-85(2001)$

[10] L. Boukhatem, D. Gaiti, G. Pujolle. A Channel Reservation Algorithm for Handover Issues in LEO Satellite Systems based on a Satellite-Fixed Cell Coverage. IEEE VTS 53rd Vehicular Technology Conference, Spring 2001, May 6 -9, 2001 Rhodes - Greece.

[11] Zhipeng Wang, Takis Mathiopoulos. Analysis and Performance Evaluation of Dynamic Channel Reservation Techniques for LEO Mobile Satellite Systems. IEEE VTS 53rd Vehicular Technology Conference, Spring 2001, May 6 -9, 2001 Rhodes Greece

[12] http://www.spaceandtech.com/spacedata/constellations/teledesic_specs.shtml

[13] A. Jamalipour. Low Earth Orbit Satellites for Personal Communications Networks, Artech House, 1997

[14] Irfan Ali, Naofal Al-Dhahir, John E. Hershey. Predicting the Visibility of LEO Satellites. IEEE Transactions on Aerospace and Electronic Systems, Vol. 35, No 4, October 1999

[15] Irfan Ali, Naofal Al-Dhahir, John E. Hershey. Doppler Characterization for LEO Satellites. IEEE Transactions on Communications, Vol. 46, No. 3, March 1998

[16] http://www.teledesic.com/newsroom/news.htm 